\title{
Feasibility of primary thermometry using refractive index measurements at a single pressure
}

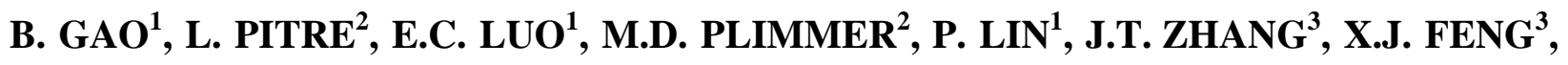 \\ Y.Y. CHEN ${ }^{1}$, F. SPARASCI ${ }^{2}$ \\ 1) Technical Institute of Physics and Chemistry, Chinese Academy of Sciences, 29 \\ Zhongguancun East Road, Haidian District, Beijing 100190, China
}

2) Laboratoire Commun de Métrologie LNE-Cnam, 61 rue du Landy, F93210 La PlaineSaint Denis, France

3) National Institute of Metrology, No. 18 Bei San Huan Dong Lu, Chaoyang District, Beijing 100029, China

E-mail (corresponding author): bgao@ mail.ipc.ac.cn 


\begin{abstract}
Refractive index gas thermometry (RIGT) uses the dependence of the relative dielectric permittivity on the density of a noble gas (He), typically measured with a temperature-controlled resonator. On an isotherm, to a good approximation, the slope of the dielectric permittivity versus pressure gives the thermodynamic temperature $T$. To measure $T$ with a low uncertainty, one must measure the absolute value of the pressure with a low uncertainty and know the compressibility of the resonator wall.
\end{abstract}

This article shows how to use RIGT in a novel way between $5 \mathrm{~K}$ and $25 \mathrm{~K}$. Instead of changing the pressure on an isotherm (J.W. Schmidt et al., Phys. Rev. Lett., 98, 254504 (2007)), a constant pressure of pure helium gas is maintained at multiple temperatures. After calibration of the resonator under vacuum at different temperatures, all thermometry is performed at a single pressure and referred to a fixed point of the International Temperature Scale of 1990 (ITS-90) (here the neon triple point at $24.5561 \mathrm{~K}$ ). The quantity that yields the temperature is the ratio of the resonance frequencies of a microwave mode in the resonator measured at the fixed point and at the unknown temperature.

This paper describes the theoretical model of Single-Pressure Refractive Index Gas Thermometry (SPRIGT) and analyses the shifts and broadenings due to non-ideal behaviour. The technique should enable a helium-based measurement of thermodynamic temperature with a resolution better than $25 \mu \mathrm{K}$ and an uncertainty of around $250 \mu \mathrm{K}$.

Keywords: Low temperature thermometry, helium gas, refractive index, microwave cavity. 


\section{Introduction}

Fundamental gas metrology, such as primary thermometry for determining the Boltzmann constant, requires that the physical property of the gas (e.g. acoustic velocity, microwave refractive index, optical Doppler width) can not only be measured precisely but also be well understood theoretically. One of the most commonly used gases is helium-4, and recent advances in the $a b$ initio calculation of its properties have made possible new variations on existing techniques. In this article we propose a variation called Single-Pressure Refractive Index Gas Thermometry (SPRIGT), which is based on the temperature and pressure dependence of the refractive index of helium. In SPRIGT, one measures a temperature by comparing the refractive index of a gas at two temperatures, one of which is known (e.g. a fixed point of the ITS-90) and the other which is to be determined. With absolute primary thermometers such as an acoustic gas thermometer (AGT) [1] or a dielectric constant-pressure gas thermometer (DCGT) [2], measurements are usually performed at several different pressures and the pressure-independent contribution is obtained by extrapolation of a function to zero pressure. By using a ratio method with measurements made under vacuum and at only a single, constant pressure, one should be able to measure temperatures about 10 times more rapidly than with either AGT or DCGT. Moreover, by using a rotating piston gauge and computer-driven servo loop, one can maintain a constant pressure for several months, if need be without the drift that typically affects other pressure sensors. The use of variable pressure gas thermometry is specified by the ITS-90 temperature scale between $5 \mathrm{~K}$ and $25 \mathrm{~K}$ but is rarely implemented due to its difficulty. We expect SPRIGT to be a welcome alternative due to its competitive accuracy and increased speed.

We begin this paper by presenting the theory relating temperature and pressure to refractive index. In particular, we show that in the ratio of refractive indices measured at different temperatures, most temperature-independent correction terms cancel out. After this we suggest 
how to determine this ratio experimentally, extract the value of the unknown temperature and estimate the associated uncertainty. We shall see the instrument is likely to have optimal performance in the range $5 \mathrm{~K}$ to $25 \mathrm{~K}$. Finally, a brief experimental procedure is outlined, together with some precautions necessary for making measurements at the envisaged level of accuracy.

\section{Temperature and pressure dependence of the refractive index}

The well-known model described here [3] is included only for clarity. Though it is not shown explicitly, almost all the variables are temperature-dependent, and many are pressure-dependent too. The refractive index $n$ relates the speed of electromagnetic waves in a medium to the value in vacuo. The index is the square root of the product of the relative electrical permittivity $\varepsilon_{r}$ and the relative magnetic permeability $\mu_{r}$ :

$$
n=\sqrt{\varepsilon_{r} \mu_{r}} .
$$

The relative permittivity $\varepsilon_{r}$ is related to the gas density $\rho$ via the Clausius-Mossotti equation:

$$
\frac{\varepsilon_{r}-1}{\varepsilon_{r}+2}=\rho A_{\varepsilon}\left(1+b_{\varepsilon} \rho+c_{\varepsilon} \rho^{2}+\ldots\right)
$$

where $A_{\varepsilon}, b_{\varepsilon}$ and $c_{\varepsilon}$ are coefficients usually determined by experiment but which, in the case of helium, can be calculated more accurately from theory $[4,5,6]$. Because ${ }^{4} \mathrm{He}$ is weakly diamagnetic, the value of $\mu_{r}$ differs slightly from unity and is obtained from the ClausiusMossotti equation for magnetic permeability:

$$
\frac{\mu_{r}-1}{\mu_{r}+2}=\rho A_{\mu}(1+\ldots) .
$$

Because the diamagnetism is so small, only a single density-dependent term is required for the accuracy sought here.

Combining equations 1 to 3 we obtain 


$$
n^{2}=\frac{1+2 A_{\varepsilon} \rho\left(1+b_{\varepsilon} \rho+c_{\varepsilon} \rho^{2}\right)\left(1+2 \rho A_{\mu}\right)}{\left(\rho A_{\mu}-1\right)\left[A_{\varepsilon} \rho\left(1+b_{\varepsilon} \rho+c_{\varepsilon} \rho^{2}\right)-1\right]} .
$$

The molar density $\rho$ depends on the pressure $p$ and temperature $T$ via the virial expansion

$$
\rho=\frac{p}{R T\left(1+B \rho+C \rho^{2}+\ldots\right)}
$$

where $R$ is the molar gas constant and $B$ and $C$ are the second and third virial coefficients.

The refractive index given by equation 4 can be estimated numerically to the required accuracy for any temperature in the interval from $5 \mathrm{~K}$ to $25 \mathrm{~K}$ and at pressures below $100 \mathrm{kPa}$. Conversely,

from a knowledge of $n^{2}$ and $p$ one can calculate the temperature $T$ by successive iteration with a relative uncertainty of $10^{-6}$ in the same range. For temperatures below $5 \mathrm{~K}$, approaching the boiling point of liquid helium $(4.2 \mathrm{~K})$, effects such as pre-condensation and liquefaction mean that more and more virial coefficients are required to calculate the temperature to such a level of accuracy. Above $25 \mathrm{~K}$ frequency noise rises: it takes longer and longer to measure the resonance frequency to the required resolution so temperature drifts degrade the accuracy

The number of terms in the expansion used to obtain $T$ is chosen such that addition of the next order term modifies its value by less than $10^{-6}$ in relative terms at $100 \mathrm{kPa}$. The values of $B(T)$ and $\mathrm{C}(T)$ and their uncertainty are taken from Shaul et al. [4], those of $\mathrm{A}_{\mu}$ and $\mathrm{A}_{\varepsilon}$ and their uncertainties from Bruch and Weinhold [5] and Lach et al. [6], and the values of $b_{\varepsilon}$ and $c_{\varepsilon}$ and their uncertainties from Rizzo et al. [7] and Gugan and Michel [8].

\section{Microwave measurement of the refractive index}

In the present case, we are interested in the refractive index of a gas in the microwave region. The resonant frequency of a gas-filled resonator depends not only on its dimensions but also on the refractive index of the gas; in one dimension, the optical length is given by the product of the 
physical length $L$, and the refractive index $n$. Put another way, electromagnetic waves travel more slowly through a medium than in vacuum so the effective wavelength is modified. The refractive index of the vacuum is unity. The deviation from unity of the refractive index of a gas mixture is a function of its pressure, temperature and composition. Unlike in the optical domain where subtle lineshape effects must be taken into account [9], in the microwave region, the refractive index is essentially density dependent. As described by Schmidt et al. (2007) [3], one can obtain the refractive index of the gas in the cavity at any temperature and pressure by comparing the resonance frequency of an electromagnetic mode with the frequency of the same mode with the cavity under vacuum, provided the change of volume due to the compression of the structure due to the pressure is taken into account. (Here we suggest using as the resonator a quasi-sphere: an almost spherical tri-axial ellipsoid [10,11]). One measures at a fixed pressure the frequency $f_{n}$ of a given mode and corrects it by an amount $\Delta f_{n}$ for two different temperatures $T$ and $T_{r e f}$. The ratio of the squares of the refractive indices at the two temperatures is related to the corrected frequencies via

$$
\frac{n^{2}(T, p)}{n^{2}\left(T_{r e f}, p\right)}=\frac{\left(\frac{\left(f_{n}+\Delta f_{n}\right)_{(T, 0)}\left(1+\frac{k_{T}(T) p}{3}\right)}{\left(f_{n}+\Delta f_{n}\right)_{T, p}}\right)^{2}}{\left(\frac{\left(f_{n}+\Delta f_{n}\right)_{(T r e f, 0)}\left(1+\frac{k_{T}\left(T_{r e f}\right) p}{3}\right)}{\left(f_{n}+\Delta f_{n}\right)_{(T r e f, p)}}\right)^{2}},
$$

where $k_{T}(T)$ and $k_{T}\left(T_{r e f}\right)$ are the isothermal compressibility of the resonator at $T$ and $T_{r e f}$. The subscript pairs $(T, 0)$ and $\left(T_{r e f}, 0\right)$ denote the temperatures $T$ and $T_{\text {ref }}$ at zero pressure, i.e. vacuum. This ratio of the two indices, one measured at a reference temperature and the other at an unknown temperature, can be determined very accurately. This is because, when a ratio is used, an exact knowledge of the corrections $\Delta f_{n}$ is not needed; provided the corrections remain 
constant with temperature they cancel out to a large extent. (This can be seen from a power series expansion of equation 6 and a consideration of the size of the remaining terms that are negligible at the level of uncertainty sought here). This means in particular that the duct and the antenna effects are absent from the ratio [10]. However, the frequencies do have to be corrected for the electrical skin depth of the electromagnetic wave, which modifies the effective radius of the resonator, since this effect varies with temperature. For the copper used in most resonators of the type suggested here $(\mathrm{CuA} 1)$, the effect at ambient temperature is related to the electrical conductivity (which itself is temperature dependent), but below $50 \mathrm{~K}$ the anomalous skin effect appears because the penetration depth for microwaves used here is a few times the crystal lattice size [12]. Here we discuss the case where the reference temperature is that of the triple point of neon $(24.5561 \mathrm{~K})$. The triple point of diatomic hydrogen at $13.81 \mathrm{~K}$ could be used as an alternative but its implementation is far more challenging than that of neon.

In microwave resonance experiments, one usually studies both transverse electric (TE) and transverse magnetic (TM) modes since they have different sensitivities to surface effects [13]. The achievable fractional frequency resolution is proportional to the half-width $g_{n}$ of a given resonance, which is a measure of the losses in the resonator. In preliminary experiments we have measured the average half-width of the mode TM11 for a quasi-sphere with a diamond turned surface. (The resonance is split into three peaks due to the deliberate non-spherical shape). We emphasize that the same quasi-sphere has already been used in the work of Sutton et al. (2011) [14] but at a higher temperature $(273.16 \mathrm{~K})$. Figure 1 gives the evolution of the half-width $g_{n}$ as a function of temperature. One can see a decrease of the slope below $50 \mathrm{~K}$ due to the anomalous skin effect and the change in the conductivity of copper. The curve gets progressively flatter at lower temperatures and the change $\Delta \mathrm{g}_{\mathrm{n}} / \Delta \mathrm{f}_{\mathrm{n}}$, which can be measured, is expected to be less than $10^{-9}$ per kelvin below $25 \mathrm{~K}$. 
A convenient way to perform experiments is to use a servo loop to lock the oscillator frequency to the maximum of a resonance. The frequency stability then depends on the shape and sharpness of the resonance, the signal-to-noise ratio (SNR), and the type of noise that predominates at the time scale under consideration. This issue has been studied for over 50 years [15] as it has a direct bearing upon the stability and ultimate accuracy of atomic clocks [16]. In the presence of white frequency noise, the frequency stability $\Delta f$ of a source servo-locked to the centre frequency $f$ of a resonance of quality factor $Q$ is characterized by the normalized Allan variance

$$
\frac{\Delta f}{f}=\frac{1}{A} \frac{1}{Q} \frac{1}{S N R} \sqrt{\frac{\tau}{t}}
$$

where $S N R$ denotes the signal-to-noise ratio for a measurement time $\tau$. For a given resonator mode of frequency $f_{n}$ and half-width $g_{n}$, the quality factor $Q$ corresponds to the ratio $\left(f_{n} / 2 g_{n}\right)$. The factor $A$ is a dimensionless constant of order unity, and $t$ is the integration time. By measuring for increasingly longer times, one can improve the frequency resolution of a measurement until other sources of noise (e.g. flicker) come into play and the Allan variance curve reaches a floor or begins to rise. A version of equation 7 can be found in Audoin and Guinot (1998) [16] for a sinusoidal response function in which the factor $A=\pi$. More generally, the value of the $A$ depends on the slope of the resonance curve and is $\approx 4$ for a Gaussian and $\approx 5$ for a Lorentzian [17]. In the experiment proposed here with a Lorentzian lineshape, a signal-to-noise ratio of 1000 can be obtained for a $2 \mathrm{~s}$ sampling time. The expected frequency resolution $(\Delta f / f)$ in the region $5 \mathrm{~K}$ to $25 \mathrm{~K}$, where $Q$ is around 160000 , will be $0.5 \times 10^{-9}$ after $4 \mathrm{~s}$. From $25 \mathrm{~K}$ up to $300 \mathrm{~K}$, the $Q$ of the resonance falls by a factor of four as the electrical resistivity rises, while all the other terms in equation (7) remain constant. In reality, it is essential to perform regular scans of the resonance line to check for any change in the width and shape. These take typically two minutes.

The change of the resonator volume due to the gas pressure is taken into account by the isothermal compressibility term $k_{T}$. Because equation 6 is a ratio, only its temperature 
dependence has any effect. The recent work of Gaiser and Fellmuth (2016) [18] gives the temperature dependence of this variable, and the authors gave a conservative upper limit of $10 \%$ for the uncertainty in $k_{T}$.

There are two other issues related to the correct measurement of pressure because the pressure gauges are located outside the resonator at room temperature. The first is the thermomolecular effect. To minimize its impact, the tube connecting the resonator to the gas handling system is tapered, with the larger diameter at the room temperature end. In this way, at the fixed pressure to be used here $(30 \mathrm{kPa})$ and at the lowest temperature $(5 \mathrm{~K})$ the molecular mean free path will always be less than the diameter of the tube, and the thermomolecular pressure shift less than 0.001 Pa. The second effect is the hydrostatic head correction, which was investigated in 1989 by Astrov and colleagues (1989) [19], and later in great detail by the LNE-Cnam group in the context of a ${ }^{3} \mathrm{He}-{ }^{4} \mathrm{He}$ vapour pressure thermometer [20].

\section{The propagated uncertainty}

Since it is not possible to obtain an analytical expression for the temperature as a function of parameters and calculate its partial derivatives, a numerical approach is used to estimate the propagated uncertainty in $T$ from the uncertainty in each parameter. First $T$ is obtained as a function of $P, k_{T}, f_{n}(P), f_{n}(0), T_{r e f}, B, C, A_{\varepsilon}, A_{\mu}$ and $b_{\varepsilon}$. Then, for each variable $X$, the calculation is repeated with $X$ replaced by $X+\sigma_{X}$, where $\sigma_{X}$ is its standard uncertainty but with all other variables constant. The total uncertainty is then evaluated as the square root of the quadratic sum of the temperature shifts due to a change in each variable taken separately. The difference between $T(X)$ and $T\left(X+\sigma_{X}\right)$ is taken for the estimation of the propagated uncertainty in the range $5 \mathrm{~K}$ to $25 \mathrm{~K}$. In this calculation, we have taken into account the resolution of the measurement in $f_{n}$ and $P$ and the uncertainty due to the imperfect knowledge of $P, T_{r e f}, B, C$ and $b_{\varepsilon}$. Due to the use 
of a ratio, variations of $A_{\varepsilon}$ and $A_{\mu}$ cancel out at the level of $10^{-12}$. The temperature variation of different contributions is shown below in Figures 2 to 4 . The uncertainty associated with the coefficients $b_{\varepsilon}, B$ and $C$ of equations 4 and 5 is shown in figure 2. The pressure measurement uncertainty contains two contributions: the absolute value of the measurement and the noise due to imperfect regulation. The uncertainties associated with the measurement and regulation of pressure are shown in figure 3. In figure 4, extra contributions due to the reference temperature, the compressibility of the resonator, and the microwave frequency measurement are added. When all uncertainty components are propagated, we obtain the graphs shown in figure 5.

The uncertainty in $T$ is linked to the working pressure chosen as shown in figure 6 . At relatively high pressures (e.g. $90 \mathrm{kPa}$ ), the effect of the uncertainty in the density virial coefficient $B$ will be the largest; at relatively low pressures (e.g. $10 \mathrm{kPa})$, the uncertainty in the frequency resolution will dominate. While the lowest uncertainty appears to be obtained at $90 \mathrm{kPa}$, there is a risk of pre-condensation at such a high pressure. A value of $30 \mathrm{kPa}$ represents a safe compromise. At this pressure the combined uncertainty for the range 5 to $25 \mathrm{~K}$ lies between $0.15 \mathrm{mK}$ and $0.25 \mathrm{mK}$, which is comparable with the accuracy provided by acoustic gas thermometry [21].

\section{Suggested experimental procedure and necessary precautions}

The purpose of this paper is to propose a method for accurate low-temperature thermometry based on relative refractive index measurements and not to describe in detail the apparatus, which is currently under development. The experimental procedure is straightforward: measure several resonance frequencies as a function of temperature, including the neon triple point 24.5561 K. The most convenient way to do this is to use a servo-loop to lock the oscillator frequency to a given resonance. To test for systematic effects, one would make measurements using both TE and TM modes [13] as well as frequent scans over line profiles (see above 
section 3). To measure temperatures, one must measure resonance frequencies with the resonator both empty and while filled with the test gas at a fixed pressure.

However, to achieve the level of accuracy illustrated by the graphs of Figures 2 to 6, particular care will be needed in key areas: pressure measurement and regulation, gas-handling, thermometry and the transport of the microwave signals. The gas pressure at $30 \mathrm{kPa}$ must be regulated to within $\pm 0.1 \mathrm{~Pa}$ and measured with an uncertainty of $30 \mathrm{ppm}$ (i.e. $1 \mathrm{~Pa}$ at $30 \mathrm{kPa}$ ). One must also estimate the hydrostatic head correction with a small uncertainty. To do so, the tubing configuration must be designed to allow calculable corrections, as in reference [20]. The procedure will involve making temperature measurements at several points along the length between resonator and pressure balance. To prevent contamination, the entire gas handling system, from the bottle of ultra-pure helium to the vacuum pumps, should have only metal tubing and all-metal joints. Moreover, one should include a cold trap at $4.2 \mathrm{~K}$ to remove any impurities. To avoid unwanted production of hydrogen within the apparatus, the use of stainless steel should be minimized.

A system similar to that used to measure the Boltzmann constant by acoustic thermometry of helium would be ideal [11], although the pressures involved here are lower. The temperature of the resonator (measured using a rhodium-iron or other suitable secondary resistance thermometer) should be regulated to within $0.01 \mathrm{mK}$, which is close to the state-of-the art. At temperatures around $24.6 \mathrm{~K}$, the thermal expansion coefficient of copper is at least 53 times lower than at room temperature [22]. This relaxes the constraint of having exactly the same temperature under vacuum and at the pressure $30 \mathrm{kPa}$ : a difference of $1 \mathrm{mK}$ would produce a relative shift of the measured frequency of only $10^{-11}$, i.e. 100 times less than the resolution. At the same time, the low thermal expansion will reduce the expansion or contraction due to a change of temperature by the same small amount. As a consequence, the speed at which the frequency changes would be limited only by the response of the gas. This will enable one to step from one temperature to another far more quickly than is possible near room temperature. Finally, particular attention should be paid to the coaxial cables used to transport the microwave signals. They also conduct heat from the hot end at room temperature to the cold end near $24 \mathrm{~K}$. 
They will cross several layers of thermal shielding and will need to be well thermalized at every stage of the cryostat before any meaningful temperature measurements can be made.

\section{Conclusion}

We have described the principle and parameters of a Single-Pressure Refractive Index Gas Thermometer (SPRIGT) for accurate temperature measurement in the range $5 \mathrm{~K}$ to $25 \mathrm{~K}$. It is based on a ratio technique that compares the refractive index of a gas at an unknown temperature and at a reference point (here the neon triple point). Due to the use of a ratio, there is no need to know the absolute value of the isothermal compressibility, $k_{T}$, of the resonator material which is perhaps the most difficult variable to estimate in RIGT and DCGT measurements; one needs only to know how it changes with temperature. The use of a ratio also alleviates the need to know the effect of the microwave correction for tube or antenna effects provided they remain constant over the temperature range in question.

Thanks to the recent reduction of the uncertainty in the ab initio calculation of the properties of helium gas, it should be possible in principle to obtain a temperature uncertainty of no more than $0.25 \mathrm{mK}$. Moreover, improvements of several important parameters seem likely in the next few years. In particular, we expect that the relative uncertainty $u_{r}\left(T_{r e f}\right)$ of the reference temperature will be reduced fourfold by careful use of acoustic or noise thermometry, and that the absolute pressure measurement uncertainty will be reduced from $33 \mathrm{ppm}$ to $5 \mathrm{ppm}$. With more refined $a b$ initio calculations, the uncertainty $u(B)$ in the coefficient $B$, will be reduced at least twofold [23]. With these expected improvements, SPRIGT should become at least as accurate as dielectric constant gas thermometers currently in use (see e.g. [24]) without the need for a low uncertainty in the absolute pressure measurement.

In addition, the device would also allow thermometry to be performed more rapidly than other instruments of comparable accuracy. Although in principle RIGT and DCGT can be performed using measurements under vacuum and at only a single pressure, in the vast majority of such experiments several pressures are required to achieve a small uncertainty. SPRIGT will be able to achieve a small uncertainty by using only measurements at vacuum and at a single pressure. 
Thus it should be possible to perform the thermometry at least 10 times faster, which would be a huge saving in time and effort.

\section{Acknowledgments}

The authors thank Dr. Robert Berg of NIST for his kind reading of the manuscript and many helpful suggestions. This work is supported financially by the National Natural Science Foundation of China (Contract No. 51627809), the international partnership program of the Chinese Academy of Sciences (Contract No. 1A1111KYSB20160017) and the EMRP project Ink2.

\section{References}

1 M.R. Moldover, R.M. Gavioso, J.B. Mehl, L. Pitre, M. de Podesta and J.T. Zhang “Acoustic gas thermometry" Metrologia 51, R1-R19 (2014).

2 C. Gaiser, T. Zandt and B. Fellmuth, "Dielectric constant gas thermometry" Metrologia 52, S217-S226 (2015).

3 J.W. Schmidt, R.M. Gavioso, E.F. May and M.R. Moldover, "Polarizability of Helium and Gas Metrology", Phys. Rev. Lett., 98, 254504 (2007) (4 pages).

4 Katherine R.S. Shaul, Andrew J. Schultz and David A. Kofke, "Path-integral Mayersampling calculations of the quantum Boltzmann contribution to virial coefficients of helium-4", J. Chem. Phys. 137, 184101 (2012) (12 pages).

$5 \quad$ L.W. Bruch and F. Weinhold, "Diamagnetism of helium", J. Chem. Phys. 113, 86678670 (2000).

6 G. Łach, B. Jeziorski and K. Szalewicz, "Radiative corrections to the polarizability of helium", Phys. Rev. Lett. 92, 233001 (2004) (4 pages).

7 A. Rizzo, C. Hättig, B. Fernandez and H. Koch, "The effect of intermolecular interactions on the electric properties of helium and argon. III. Quantum statistical calculations of the dielectric second virial coefficients", J. Chem. Phys. 117, 26092618, (2002). 
8 D. Gugan and G.W. Michel, "Dielectric constant gas thermometry from 4.2 to 27.1 K” Metrologia 16, 149-167, (1980).

9 A. Corney “Atomic and Laser Spectroscopy” (Oxford, 1977).

10 R.J. Underwood, J.B. Mehl, L. Pitre, G. Edwards, G. Sutton and M. de Podesta, "Waveguide effects on quasispherical microwave cavity resonators", Meas. Sci. Technol. 21, 075103 (2010) (17 pages).

11 L. Pitre, L. Risegari, F. Sparasci, M.D. Plimmer, M.E. Himbert, P.A. Giuliano Albo, "Determination of the Boltzmann constant k from the speed of sound in helium gas at the triple point of water", Metrologia 52(5), S263-S273 (2015).

12 B. Podobedov, "Resistive wall wakefields in the extreme anomalous skin effect regime”, Phys. Rev. Spec. Top. - Accel. Beams, 12, 044401 (2009) (10 pages).

13 E.F. May, L. Pitre, J.B. Mehl, M.R. Moldover, J.W. Schmidt, "Quasi-Spherical Cavity Resonators for Metrology Based on the Relative Dielectric Permittivity of Gases”, Review of Scientific Instruments 75,3307-3317 (2004).

14 G. Sutton, R. Underwood, L. Pitre, M. de Podesta and S. Valkiers, "Acoustic resonator experiments at the triple point of water: first results for the Boltzmann constant and remaining challenges", Int. J. Thermophys. 31, 1310-1346 (2011).

15 D.W. Allan, "Statistics of Atomic Frequency Standards", Proc. IEEE, 54, 221-230 (1966).

16 C. Audoin and B. Guinot "Les fondements de la mesure du temps" (Masson 1998) Ch. 6. Book available in English as "The Measurement of time: Time, frequency and the atomic clock" (Cambridge, 2001).

17 P. Juncar, private communication (2016). 
18 C. Gaiser and B. Fellmuth, "Method for extrapolating the compressibility data of solids from room to lower temperatures", Phys. Status Solidi B. 253, 1549-1556 (2016).

19 D.N. Astrov, L.B. Beliansky, Y.A. Dedikov, S.P. Polunin and A.A. Zakharov, "Precision gas thermometry between $2.5 \mathrm{~K}$ and $308 \mathrm{~K}$ " Metrologia 26, 151-166 (1989).

20 F. Sparasci, L. Pitre, D. Truong, L. Risegari and Y. Hermier, "Realization of a ${ }^{3} \mathrm{He}-$ ${ }^{4} \mathrm{He}$ Vapor-Pressure Thermometer for Temperatures between $0.65 \mathrm{~K}$ and $5 \mathrm{~K}$ at LNECNAM", Int. J. Thermophys. 32, 139-152 (2011).

21 L. Pitre, M.R. Moldover and W.L. Tew, "Acoustic thermometry: new results from $273 \mathrm{~K}$ to $77 \mathrm{~K}$ and progress towards 4 K”, Metrologia 43, 142-162 (2006).

22 P.M.C. Rourke and K.D. Hill, "Progress Toward Development of Low-Temperature Microwave Refractive Index Gas Thermometry at NRC", Int. J. Thermophys. 36, 205-228 (2015).

23 B. Jeziorsky, private communication (2016).

24 C. Gaiser, B. Fellmuth and N. Haft "Dielectric-Constant Gas-Thermometry Scale from $2.5 \mathrm{~K}$ to $36 \mathrm{~K}$ Applying ${ }^{3} \mathrm{He},{ }^{4} \mathrm{He}$, and Neon in Different Temperature Ranges", Int. J. Thermophys. 31, 1428-1437 (2010). 


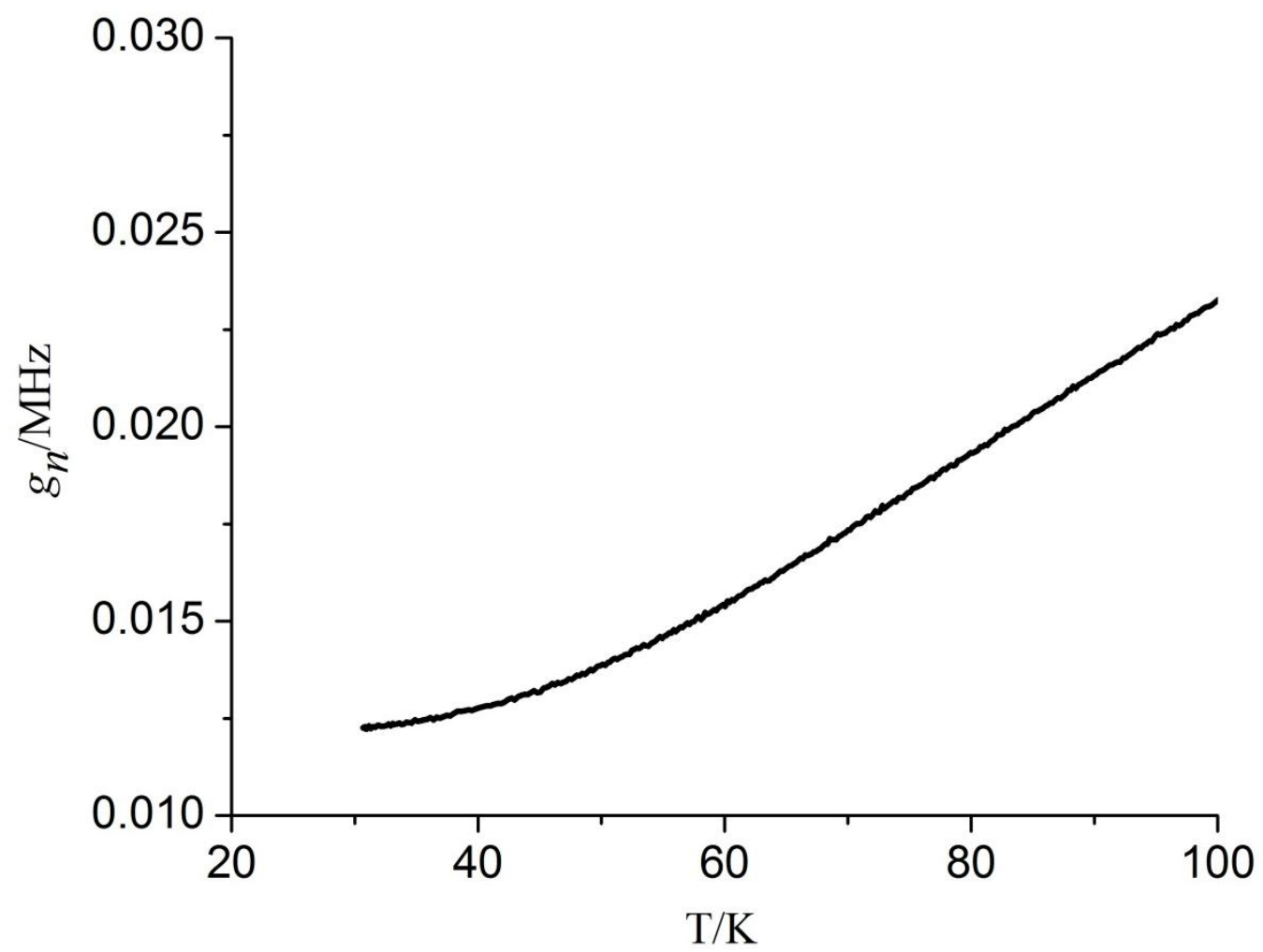

Figure 1. The half-width $g_{n}$ of a microwave resonance in a quasi-sphere as a function of temperature in the range $30 \mathrm{~K}-100 \mathrm{~K}$. The half-width is even smaller below $30 \mathrm{~K}$. 


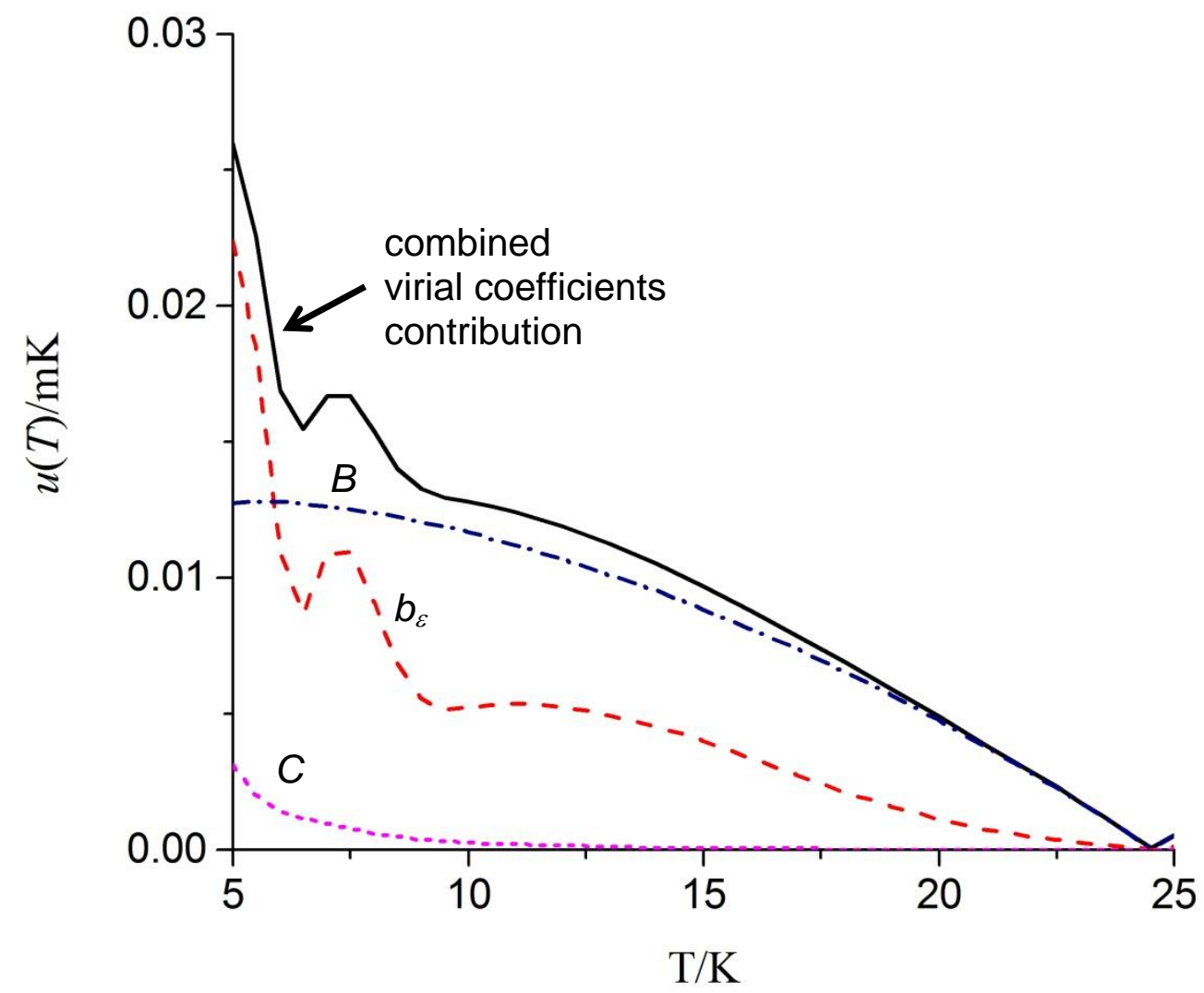

Figure 2. Expected contributions to the uncertainty due to the virial coefficients $B, C$, and $b_{\varepsilon}$, for temperature measurements referred to the triple point of neon $(24.6 \mathrm{~K})$. The hump near $7 \mathrm{~K}$ is due to values of uncertainties for $B$ given in the literature [2]. Key: ----- dielectric virial coefficient $b_{\varepsilon}$ : ------- virial coefficient $C, \cdot-\cdot-\cdot$-virial coefficient $B ;-$ combined uncertainty due to virial coefficients. 


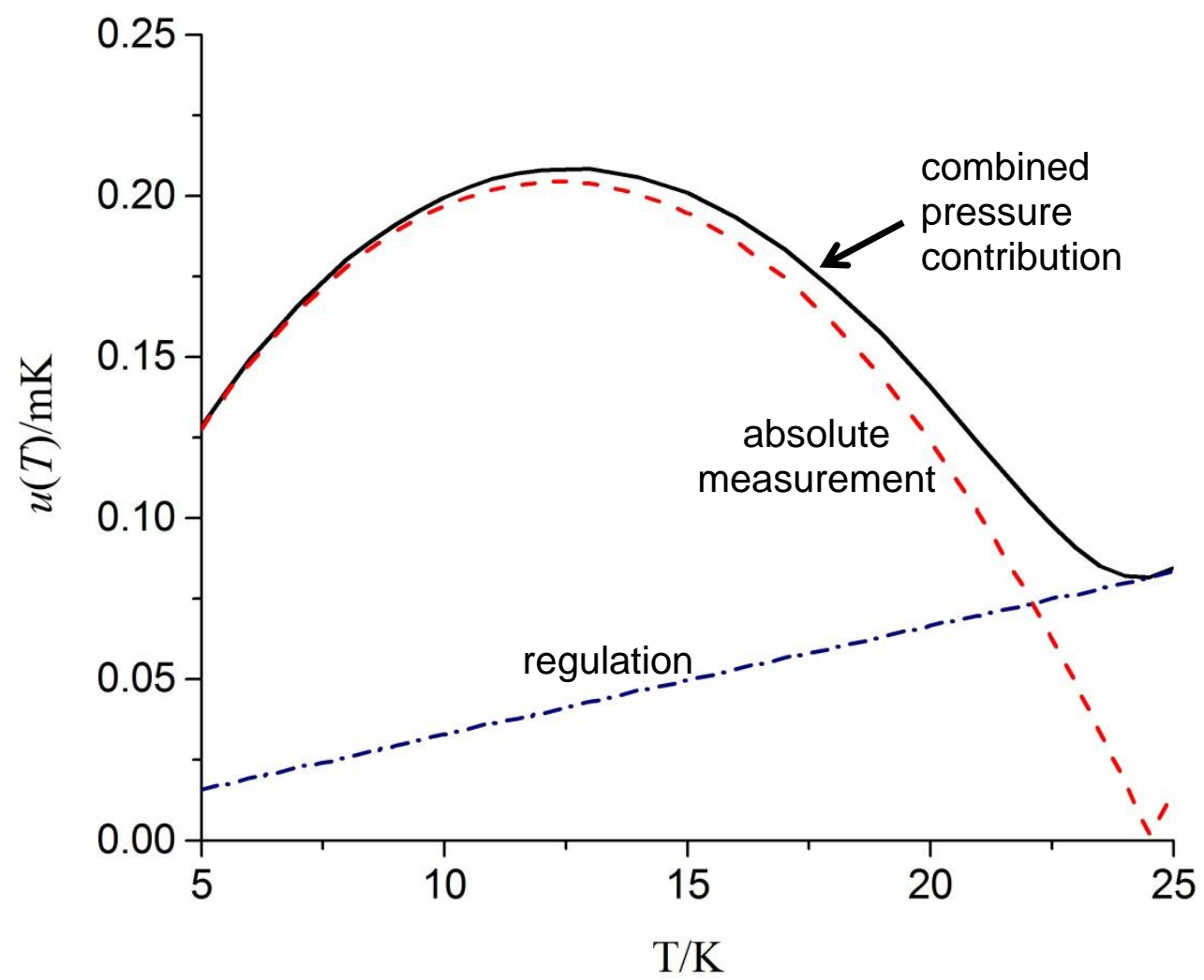

Figure 3. Expected contributions to the uncertainty due to pressure at $30 \mathrm{kPa}$, for temperature measurements referred to the triple point of neon $(24.6 \mathrm{~K})$. Key: ----- absolute pressure measurement $: \cdot-\cdot-\cdot-$ pressure regulation $;-$ combined uncertainty due to pressure. 


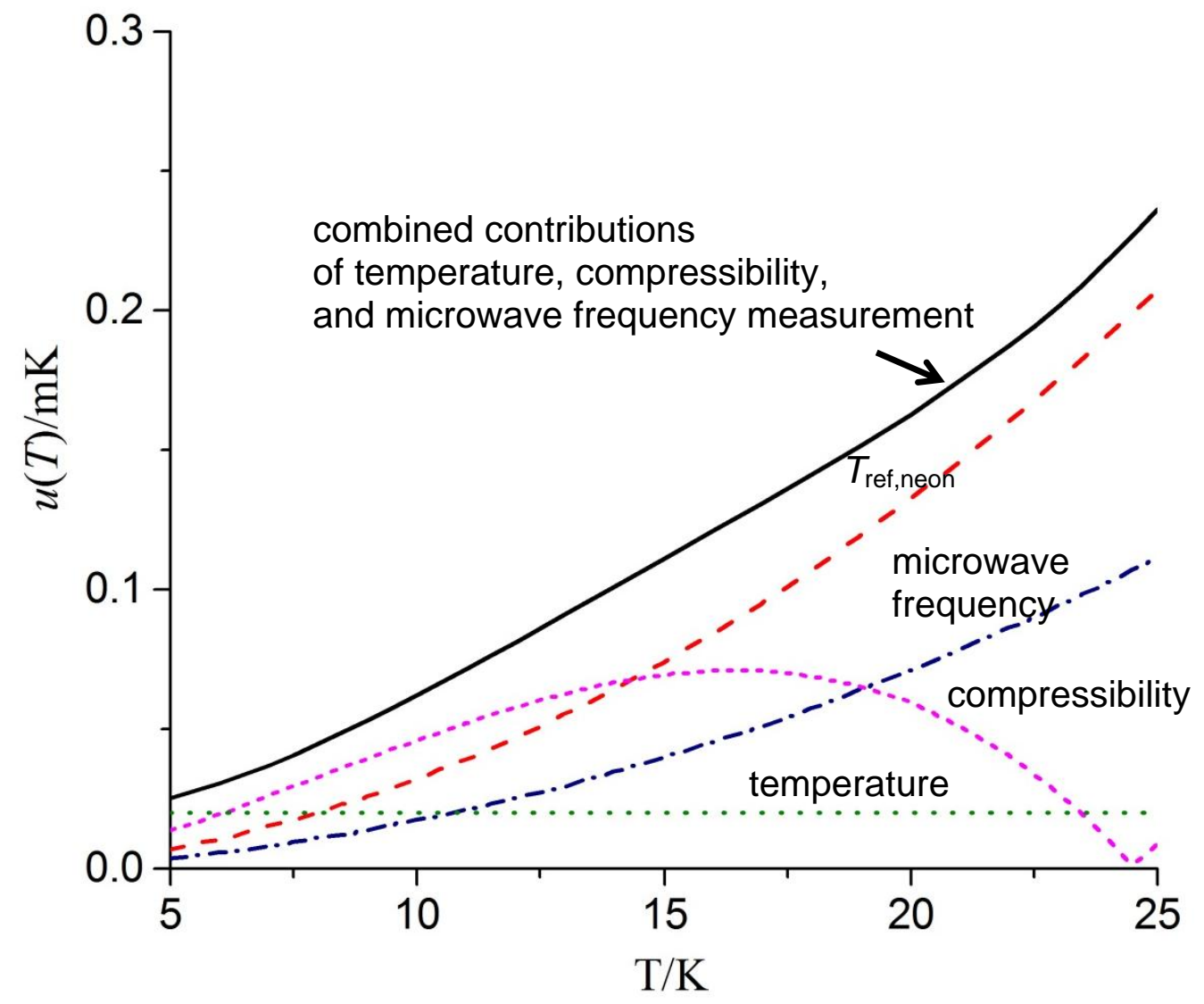

Figure 4. Expected contributions to the uncertainty due to temperature, the compressibility of the resonator, the microwave frequency, and the reference temperature, for temperature measurements referred to the triple point of neon $(24.6 \mathrm{~K})$. Key: $-----T_{\text {ref,neon }}:-------$ compressibility from Gaiser et al.[13 ], $\cdot-\cdot-\cdot-$ microwave frequency measurement $; \cdots \cdots$ temperature control; $—$ combined uncertainty due to measurement. 


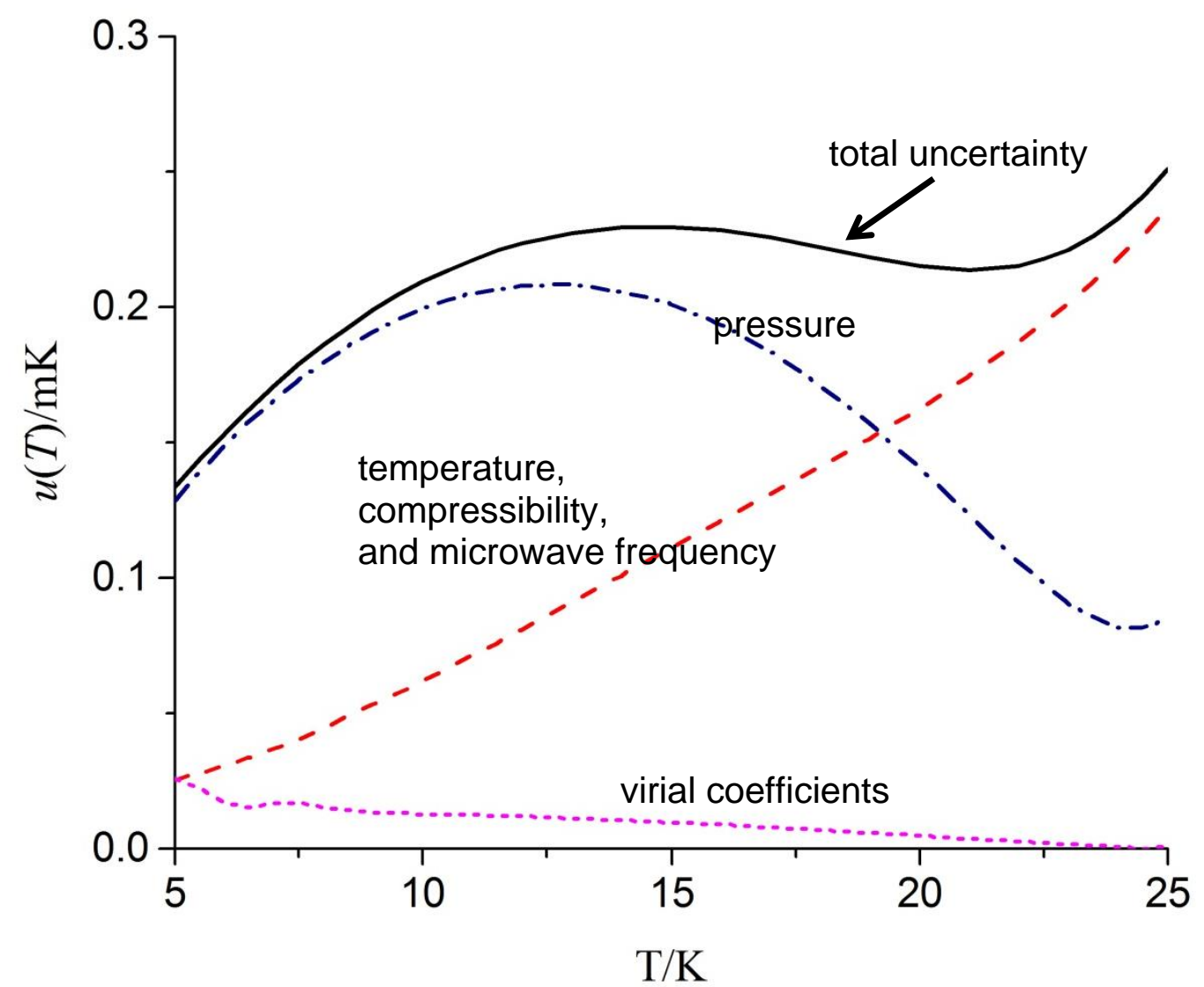

Figure 5. Total expected uncertainty, from Figures 2-4 at $30 \mathrm{kPa}$, in temperature measurements referred to the triple point of neon $(24.6 \mathrm{~K})$. At temperatures below $20 \mathrm{~K}$, the dominant contribution is due to the pressure measurement.

Key :-- - - combined measurement : ------- combined virial, $\cdot-\cdot-\cdot-$ combined pressure ; combined uncertainty. 


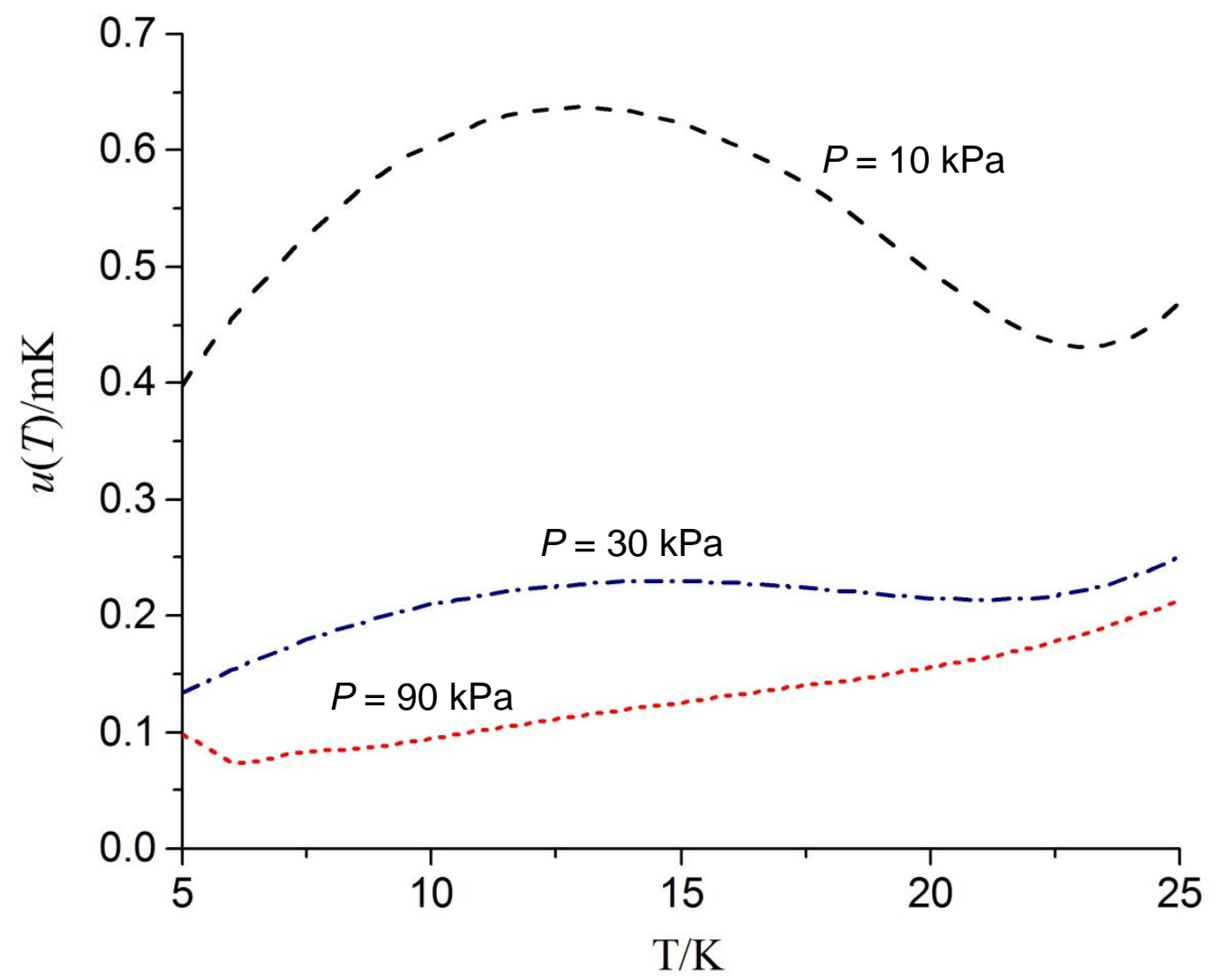

Figure 6. Total uncertainty expected in temperature measurements referred to the triple point of neon $(24.6 \mathrm{~K})$, for fixed gas pressures in the range $10 \mathrm{kPa}$ to $90 \mathrm{kPa}$. Key:---- $\mathrm{P}=10 \mathrm{kPa}$; $\cdot-\cdot-\mathrm{P}=30 \mathrm{kPa}$; ------ $\mathrm{P}=90 \mathrm{kPa}$. 


\section{Figure Captions}

\section{Figure 1}

The half-width $g_{n}$ of a microwave resonance in a quasi-sphere as a function of temperature in the range $30 \mathrm{~K}-100 \mathrm{~K}$. The half-width is even smaller below $30 \mathrm{~K}$.

\section{Figure 2}

Expected contributions to the uncertainty due to the virial coefficients $B, C$, and $b_{\varepsilon}$, for temperature measurements referred to the triple point of neon $(24.6 \mathrm{~K})$. The hump near $7 \mathrm{~K}$ is due to values of uncertainties for $B$ given in the literature [2]. Key: - - - - dielectric virial coefficient $b_{\varepsilon}$ : ------- virial coefficient $C, \cdot-\cdot-\cdot-$ virial coefficient $B ;-$ combined uncertainty due to virial coefficients.

\section{Figure 3}

Expected contributions to the uncertainty due to pressure at $30 \mathrm{kPa}$, for temperature measurements referred to the triple point of neon $(24.6 \mathrm{~K})$. Key: ----- absolute pressure measurement: $\cdot-\cdot-\cdot-$ pressure regulation ;_—_ combined uncertainty due to pressure.

\section{Figure 4}

Expected contributions to the uncertainty due to temperature, the compressibility of the resonator, the microwave frequency, and the reference temperature, for temperature measurements referred to the triple point of neon $(24.6 \mathrm{~K})$. Key :-- - - $\mathrm{T}_{\text {ref,neon }}:-------$ compressibility from Gaiser et al. $[13], \cdot-\cdot-\cdot-$ microwave frequency measurement $; \cdots \cdots$ temperature control; $\longrightarrow$ combined uncertainty due to measurement.

\section{Figure 5}


Total expected uncertainty, from Figures $2-4$ at $30 \mathrm{kPa}$, in temperature measurements referred to the triple point of neon $(24.6 \mathrm{~K})$. At temperatures below $20 \mathrm{~K}$, the dominant contribution is due to the pressure measurement.

Key :-- - - combined measurement : ------- combined virial, $\cdot-\cdot-\cdot-$ combined pressure ; combined uncertainty.

\section{Figure 6}

Total uncertainty expected in temperature measurements referred to the triple point of neon (24.6

$\mathrm{K}$ ), for fixed gas pressures in the range $10 \mathrm{kPa}$ to $90 \mathrm{kPa}$. Key : $-----\mathrm{P}=10 \mathrm{kPa} ;-\cdot-\cdot-$ $\mathrm{P}=30 \mathrm{kPa} ;------\mathrm{P}=90 \mathrm{kPa}$. 